\title{
The Functionality of Facial Appearance and Its Importance to a Korean Population
}

\author{
Young Jun Kim ${ }^{1}$, Jang Wan Park ${ }^{1}$, Jeong Min Kim ${ }^{1}$, Sun Hyung Park ${ }^{1}$, Jae Ha Hwang ${ }^{1}$, \\ Kwang Seog Kim ${ }^{1}$, Sam Yong Lee ${ }^{1}$, Jun Ho Shin ${ }^{2}$ \\ Departments of ${ }^{1}$ Plastic and Reconstructive Surgery and ${ }^{2}$ Preventive Medicine, Chonnam National University Medical School, Gwangju, \\ Korea
}

\begin{abstract}
Background Many people have an interest in the correction of facial scars or deformities caused by trauma. The increasing ability to correct such flaws has been one of the reasons for the increase in the popularity of facial plastic surgery. In addition to its roles in communication, breathing, eating, olfaction and vision, the appearance of the face also plays an important role in human interactions, including during social activities. However, studies on the importance of the functional role of facial appearance. As a function of the face are scare. Therefore, in the present study, we evaluated the importance of the functions of the face in Korea.

Methods We conducted an online panel survey of 300 participants (age range, 20-70 years). Each respondent was administered the demographic data form, Facial Function Assessment Scale, Rosenberg Self-Esteem Scale, and standard gamble questionnaires.

Results In the evaluation on the importance of facial functions, a normal appearance was considered as important as communication, breathing, speech, and vision. Of the 300 participants, $85 \%$ stated that a normal appearance is important in social activities.

Conclusions The results of this survey involving a cross-section of the Korean population indicated that a normal appearance was considered one of the principal facial functions. A normal appearance was considered more important than the functions of olfaction and expression. Moreover, a normal appearance was determined to be an important facial function for leading a normal life in Korea.
\end{abstract}

Keywords Face / Social perception / Adult
Correspondence: Kwang Seog Kim Department of Plastic and Reconstructive Surgery, Chonnam National University Medical School, 42 Jebong-ro, Dong-gu, Gwangju 501-757, Korea

Tel: +82-62-220-6363

Fax: +82-62-227-1639

E-mail: pskim@chonnam.ac.kr

No potential conflict of interest relevant to this article was reported.

Received: 14 Mar 2013 • Revised: 17 Jul 2013 • Accepted: 18 Jul 2013

pISSN: 2234-6163 • elSSN: 2234-6171 • http://dx.doi.org/10.5999/aps.2013.40.6.715 • Arch Plast Surg 2013;40:715-720

\section{INTRODUCTION}

All human faces are unique and contribute to individual identity. Moreover, the face is one of the most fundamental parts of the body for self-recognition. Vision, hearing, olfaction, eating, and breathing are considered the primary functions of the face. In addition, the face is important for expression. People who experience facial deformities due to trauma or burns possess a negative self-image compared to those with normal faces [1]. "Function" has been defined as "taking a role or action" or "the natural action or intended purpose of a person or thing in a specific role" [2]. With regard to function, it is certain that a normal appearance plays a significant role in an individual's life. Moreover, there is an increasing social recognition that beauty is

Copyright $(\odot 2013$ The Korean Society of Plastic and Reconstructive Surgeons

This is an Open Access article distributed under the terms of the Creative Commons Attribution Non-Commercial License (http://creativecommons.org/

licenses/by-nc/3.0/) which permits unrestricted non-commercial use, distribution, and reproduction in any medium, provided the original work is properly cited.

www.e-aps.org 
a characteristic that can be acquired.

The number of individuals undergoing plastic surgery has also been increasing, which indicates an increase in people's desire to improve their appearance [3]. Although certain studies in Korea have assessed the effect of plastic surgery on health [3-5], research on the relative importance of a normal appearance compared to other facial functions is scarce.

In the present study, we investigated the relative importance and the awareness of the various functions of the face as perceived by Korean people through a cross-sectional cohort study based on demographic statistics. It specifically addressed the following questions: 1) What is the fundamental function of the face in normal individuals? and 2) How do individuals rate the importance of each facial function relative to the other facial functions?

\section{METHODS}

\section{Design}

A specifically designed questionnaire was developed in the present study to investigate facial characteristics and functions. The measurements included the degree of risk that was acceptable for achieving a normal appearance and the relative importance of the facial functions. The questionnaire was based on the study by Borah and Rankin [6], but adapted for use in the Korean population.

\section{Population}

A total of 300 Korean individuals participated in the online panel survey. The average age of the participants was 42.8 years. The data were collected via the internet from individuals residing in different parts of the country. Informed consent was obtained from participants prior to initiation of the study. The questionnaire was administered in the form of a presentation. The next question was asked to the participant only after the answer to the previous question was obtained. Most of the questions had answers that included multiple choices, and the respondents answered these questions by clicking on the appropriate choice. Some questions asked for a ranking of the choices, whereas others were short open-ended questions. Outliers were eliminated for consistency and fidelity. A quota-sampling design was applied to the survey based on the age distribution of the Korean population. The size of each age group was determined based on sample extraction [7]. In addition, gender, age, and the degree of education were normalized for the survey. Thus, we assigned the respondents to 5 groups, each consisting of 40 to 72 individuals, according to their age.

\section{Materials}

The questionnaire comprised 4 categories: the Demographic Data Form, the Facial Function Assessment Scale, a standard gamble questionnaire, and the Rosenberg Self-Esteem Scale. A demographic survey was initially conducted, whereby items such as gender, age, and place of residence were recorded. If the participant fulfilled the inclusion criteria, the participant could then proceed to the following series of questions. Next, the data survey collected information on religion, job type, highest educational degree, presence of facial deformity, and history of facial surgery.

\section{Facial Function Assessment Scale}

The Facial Function Assessment Scale was designed to investigate the relative importance of each function of the face, whether a normal appearance was recognized as a facial function, and the importance of a normal appearance. The concept of "normal appearance" was explained to the respondents who were administered the Facial Function Assessment Scale questionnaire. Normal appearance was defined as a natural appearance that does not cause any negative reaction from other individuals during an interaction (Fig. 1). For example, changes in the facial skin or contours caused by an accident or an abnormality were included in the non-normal appearance category. After the participants understood the explanation, they would then proceed to the next question in the survey. In the first question of the Facial Function Assessment Scale, the respondents were asked about basic functions necessary for a normal face (Fig. 2). Examples of functions included chewing, communication, nose breathing, smiling, normal appearance, symmetry, frowning, sensation, and beauty; multiple answers were permitted. The percentage of individuals opting for each function was calculated, and the functions were listed in order of preference. In the next question, the respondents were asked about the relative importance of the facial functions. Fig. 3 illus-

\section{Fig. 1. Concept of normal appearance}

The concept of normal appearance as a facial function was explained to the respondents.

"Normal appearance" mentioned in this questionnaire is not a notion of beauty, but it is a natural appearance that does not show a sense of incon gruity or evoke a negative feeling in others.

For example, a negative image resulting from changes of facial skin or sh ape due to an accident or deformity can be considered to be "not a stand ard normal appearance."

Do you fully understand the notion of the above "normal appearance"? 


Fig. 2. First question in the facial function survey
\begin{tabular}{|l|l|l|} 
Question inquiring about the most important facial function among \\
individuals with a normal facial appearance.
\end{tabular}
$\begin{aligned} & \text { What functions are required to satisfy a normal face? (Multiple answers } \\
& \text { permitted) }\end{aligned}$
\begin{tabular}{|l|l|l|l}
\hline$\square$ Chewing & Communication \\
\hline$\square$ Nose breathing & $\square$ Smiling \\
\hline$\square$ Normal appearance & $\square$ Symmetry \\
\hline$\square$ Frowning & $\square$ Sensation \\
\hline$\square$ Beauty & \\
\hline
\end{tabular}

\section{Fig. 3. Second question in the facial function survey}

Measurement of the relative importance of the facial functions.

These are questions to evaluate the relative importance among facial functions. Please mark the importance of each function relative to the importance of other functions.

(Please mark the importance from No. 1 to No. 7--No. 1 is the most

important and No. 7 is the least important.)

\begin{tabular}{|l|c|c|c|c|c|c|c|}
\hline \multicolumn{1}{|c|}{ Most important -------- Middle -------- Least important } \\
\hline Breathing & 1 & 2 & 3 & 4 & 5 & 6 & 7 \\
\hline Vision & $\square$ & $\square$ & $\square$ & $\square$ & $\square$ & $\square$ & $\square$ \\
\hline Speech & $\square$ & $\square$ & $\square$ & $\square$ & $\square$ & $\square$ & $\square$ \\
\hline Eating & $\square$ & $\square$ & $\square$ & $\square$ & $\square$ & $\square$ & $\square$ \\
\hline Appearance & $\square$ & $\square$ & $\square$ & $\square$ & $\square$ & $\square$ & $\square$ \\
\hline Expression & $\square$ & $\square$ & $\square$ & $\square$ & $\square$ & $\square$ & $\square$ \\
\hline Olfaction & $\square$ & $\square$ & $\square$ & $\square$ & $\square$ & $\square$ & $\square$ \\
\hline
\end{tabular}

trates the question used to elicit the relative importance of facial functions. A number scoring system was applied, ranging from 1 to 7 , with 1 indicating the "most important" to 7 indicating the "least important." The average score for each function was calculated, and the score of each function was evaluated based on its relative difference from the score of 1 (Fig. 3). In the third question, the respondents were asked whether certain surgeries were functional or aesthetic (Fig. 4). In the fourth question, respondents were asked whether there is an association between normal appearance and social activity (Fig. 5).

\section{Standard gamble questionnaire}

The standard gamble questionnaire evaluated the extent of the risk of death that the individuals were willing to accept in order to achieve a normal facial appearance [8]. For example, if the respondent recorded an answer of $40 \%$, it implied that the respondent would undertake a $40 \%$ risk of death to achieve a normal facial appearance through surgery.

\section{Rosenberg Self-Esteem Scale}

The Self-Esteem Scale developed by Rosenberg [9] in 1965 was used for self-esteem measurements in the present study. The Rosenberg Self-Esteem Scale is similar to other social survey questionnaires. It comprises a 10-item Likert-type scale, with

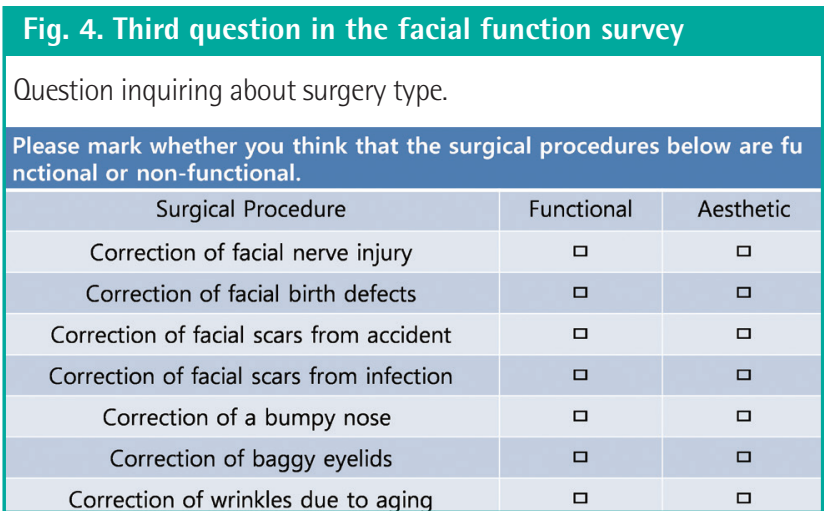

Fig. 5. Fourth question in the facial function survey

(A) Question inquiring if normal appearance is important for normal social activity. (B) Question inquiring if normal appearance is related for normal social activity.

Please read the question carefully and then select the appropriate ans wer.

A normal appearance is important for normal social activity in Korea.

\begin{tabular}{l} 
Yes, it is important \\
\hline$\square$ \\
No, it is not important
\end{tabular}

Please read the question carefully and then select the appropriate ans wer.

A normal appearance is related to normal social activity in Korea.

\begin{tabular}{l|l}
\hline$\square$ & Yes, it is related. \\
\hline$\square$ & No, it is not unrelated.
\end{tabular}

items scored on a 4-point scale, ranging from "strongly agree" to "strongly disagree." Five of the items have positively worded statements and 5 have negatively worded statements. The scale measures self-esteem by asking the respondents to reflect on their feelings at that time. The English version of the Rosenberg Self-Esteem Scale was first translated to Korean, and the Korean version was then back-translated to English. The 2 English versions were then compared in order to validate the accuracy of the translated version, and changes were made, as required, to ensure that the original meaning was communicated. The final translated version of the Rosenberg Self-Esteem Scale was used to measure and evaluate the score, with a range from 20 to 40 indicating a person with high self-esteem. Survey error was minimized by using the same score range data. The respondents completed the questionnaire in approximately 10 minutes.

\section{Pilot study and statistical analysis}

A paper based pilot test was conducted on 200 students (age range, 22-26 years) of a large national university medical school. The participants were given privacy and answered the questions in a controlled environment. The data analysis yielded a score of 
Table 1. Subjects' assessment of the importance of basic functions required to satisfy a normal face $(n=300)$

\begin{tabular}{|lc|}
\hline Function & $\%$ \\
\hline Normal appearance & 75.0 \\
Communication & 66.0 \\
Smiling & 63.0 \\
Chewing & 48.7 \\
Sensation & 43.0 \\
Breathing & 41.3 \\
Symmetry & 41.3 \\
Beauty & 13.0 \\
Frowning & 12.7 \\
\hline
\end{tabular}

\section{Table 2. Subjects' rank order of facial functions $(n=300)$}

\begin{tabular}{|lc|}
\hline Facial functions & Score $^{\text {a) }}$ \\
\hline Communication & 2.66 \\
Breathing & 2.78 \\
Eating & 2.82 \\
Vision & 2.92 \\
Normal appearance & 2.97 \\
Expression & 3.01 \\
Olfaction & 3.20 \\
\hline a)1, most important; 7, least important. & \\
\hline
\end{tabular}

$>0.6$ using Cronbach's coefficient alpha value, which was considered to be significant and to indicate reliability. The collected data were analyzed using Excel and PASW ver. 18 (SPSS Inc., Chicago, IL, USA) software. Facial functions and their relative importance were measured using frequency analysis.

\section{RESULTS}

\section{Demographic data}

In total, 300 respondents completed the survey. The survey was conducted using demographic distribution provided by the Korea Statistics Bureau [10]. The age of the respondents ranged from 20 to 70 years, and the average age was 42.8 years. The ratio of male to female respondents was 1:1. With regard to education, $28 \%$ of all of the respondents had only a high school diploma, 63 percent had a bachelor's degree, and 9 percent had a master's degree or higher.

\section{Facial Function Assessment Scale}

For the first question of the Facial Function Assessment Scale questionnaire, normal facial appearance, communication, and smiling were found to be the most commonly reported items. The beauty function of the face and the wrinkle function of the forehead were the responses that were chosen the least (Table 1). The second question (Fig. 3) measured the relative importance of the facial functions. In this questionnaire, communica-
Table 3. Subjects' assessment of surgical procedures as functional or aesthetic $(n=300)$

\begin{tabular}{|lcc|}
\hline Surgical procedure & Functional (\%) & Aesthetic (\%) \\
\hline Correction of facial nerve injury & 97 & 3 \\
Correction of facial birth defects & 89 & 11 \\
$\begin{array}{l}\text { Correction of facial scars from an } \\
\quad \text { accident }\end{array}$ & 67 & 33 \\
$\begin{array}{l}\text { Correction of facial scars from an } \\
\text { infection }\end{array}$ & 71 & 29 \\
Correction of a bumpy nose & 43 & 57 \\
Correction of baggy eyelids & 31 & 69 \\
Correction of wrinkles due to aging & 9 & 91 \\
\hline
\end{tabular}

tion was ranked the most important function, with 2.66 points. Other highly ranked responses included breathing (score, 2.78) and eating (score, 2.82). A normal appearance of the face ranked fifth in importance. The least important items included emotional expression (score, 3.01) and olfaction (score, 3.20) (Table 2). In the third question (Fig. 4), the patients were asked whether certain surgeries were functional or aesthetic (Table 3). Most subjects (97\%) considered surgery to normalize facial nerve injury to be functional. Moreover, a large proportion of subjects (89\%) reported that surgery to normalize the appearance of a congenital facial disfigurement was functional. Sixtyseven percent of the respondents agreed that surgery to normalize the appearance of facial scars resulting from accidents was functional, as compared with $33 \%$ of subjects who considered this type of surgery to be aesthetic. In the fourth question, the statement, "Normal facial appearance is important for social activity in Korea," was accepted by $85 \%$ of the participants and was rejected by $15 \%$ (Fig. 5). The next statement, "A normal appearance is related to normal social activity in Korea," was accepted by $83 \%$ of the participants and rejected by $17 \%$.

\section{Standard gamble questionnaire}

The results of the standard gamble questionnaire showed that the average acceptable risk of death for obtaining a normal facial appearance was $34 \%$.

\section{Rosenberg Self-Esteem Scale}

None of the respondents of the Rosenberg Self-Esteem Scale scored less than 20; the average score of the respondents was 28.98. The survey results of the respondents indicated that they had a high level of self-esteem and a positive attitude in general.

\section{DISCUSSION}

In the present study, we aimed to investigate the general consensus regarding the normal functions of the human face through 
an online panel survey. This study involving a cross-section of the Korean population indicated that normal appearance is more important than beauty itself. Further, a normal facial appearance is considered to be one of the important functions of the face. This function is also considered an important factor in daily life and activities.

An interesting fact raised by this study is that the normal appearance of the face was considered an important factor for social activity. According to a previous study, individuals without a normal facial appearance possessed a negative self-image. This trend of thinking negatively influenced their social activity. In addition, that study indicated that people do not have a favorable impression of an individual with an abnormal facial appearance caused by injury or burns, and may underestimate that individual's social ability [1]. In the present study, a normal facial appearance was found to be one of the important functions of the face, with a $75 \%$ positive response rate in the questionnaire. However, facial beauty was not considered one of the important functions of the face, with a $13 \%$ positive response rate. This indicates that only a small proportion of individuals consider facial beauty to be a facial function.

The findings of the present study performed in Korea were compared to those of the study conducted by Borah and Rankin [6] in the United States. A normal facial appearance ranked fifth with regard to the importance of facial functions in both studies. Both studies showed that the functions directly associated with life (breathing and eating) were considered the most important, followed by the sense-related functions (vision and speech). Basic values as well as living patterns appear to be similar in the two countries, part of the reason for which could be the influence of mass media, such as the internet.

In the Borah and Rankin [6] study, 57\% agreed that normal facial appearance is a basic facial function; in the present study in Korea, $75 \%$ of the participants considered normal facial appearance to be a basic facial function. Due to the differences of the investigation methods and questionnaires, the significance of the difference between these two values was difficult to determine. However, while general life-related functions were considered relatively important in the study in the United States, normal appearance was considered to be relatively important in the present Korean investigation. We believe that the Korean participants responded to the questionnaire while considering the impact of a normal facial appearance in social life; moreover, Korean individuals appear not to associate life-related functions with basic facial functions. Furthermore, in the study by Borah, $43 \%$ of participants considered beauty to be a basic facial function, whereas only $13 \%$ indicated this answer in the present study; there is a marked difference in these values, and this point of view was least popular in the present study. In addition, the proportion of individuals who considered surgical correction of a bumpy nose (84\%) and droopy eyelids (87\%) as aesthetic procedures in the Borah study was markedly higher than the those who held similar opinions in the present study. This indicates that normal appearance is considered to have a more comprehensive meaning to Korean individuals, and that Koreans are relatively tolerant of aesthetic surgery.

The standard gamble questionnaire results showed that an average of $34 \%$ risk of death was acceptable to Korean individuals for achieving a normal facial appearance; however, the corresponding value was only $7 \%$ in the study of Borah on American individuals. Thus, as also indicated by the preceding questions, normal appearance is considered to be more important to Korean individuals.

One limitation of this study is that it involved an online survey method, which could have affected its validity or reliability. However, there are also several advantages to an internet-based survey. The online survey takes a relatively short time, is less expensive, is easy to answer, reduces interviewer-induced errors, and also applies more diverse multimedia techniques than traditional surveys. Moreover, the reliability of online surveys is reported to be similar to that of offline surveys $[11,12]$. Thus, the online panel survey is a credible method, but requires a wellestablished survey design based on representative samples [12]. The relatively small number of respondents with an advanced age is another limitation of the present study. In addition, the proportion of individuals with a bachelor's degree or a higher degree was higher in the study sample than in the general population. These sampling problems limit the potential to expand these results to the general Korean population.

In the Korean population, we noted that a normal appearance was considered to be one of the principal facial functions. Moreover, a normal appearance was considered to be an important facial function in order to lead a normal life in Korea. Therefore, plastic surgeons who treat facial deformities or facial injuries through reconstruction should consider not only functional aspects but also facial appearance, which itself has functions for identity and social interaction.

\section{REFERENCES}

1. Rankin M, Borah GL. Perceived functional impact of abnormal facial appearance. Plast Reconstr Surg 2003;111:2140-6.

2. The Collins English dictionary: definition of "function" [Internet]. London: Collins; c2013 [cited 2013 Aug 20]. Available from: http://www.collinsdictionary.com/dictionary/ english/function?showCookiePolicy=true. 
3. Ahn JH, Kim YU, Paik MH, et al. The psychological aspect in plastic and reconstructive surgery patients (II). J Korean Soc Plast Reconstr Surg 1993;20:945-68.

4. Bang SI, Kim HJ, Song YH, et al. The psychiatric and psychological characteristics of cosmetic surgery patients and the effect of cosmetic surgery. J Korean Soc Aesthetic Plast Surg 2001;7:1-7.

5. Han SJ, Ahn JH, Baik MH, et al. The psychological aspect in plastic surgery patients (I). J Korean Soc Plast Reconstr Surg 1991;18:1015-22.

6. Borah GL, Rankin MK. Appearance is a function of the face. Plast Reconstr Surg 2010;125:873-8.

7. Cohen J. Statistical power for the behavioral sciences. New York: Academic Press; 1977.

8. Littenberg B, Partilo S, Licata A, et al. Paper standard gamble: the reliability of a paper questionnaire to assess utility. Med Decis Making 2003;23:480-8.

9. Rosenberg M. Society and the adolescent self-image. Princeton, NJ: Princeton University Press; 1965.

10. National Institutes of Statistics Korea. Population census data (2010) [Internet]. Dajeon: Statistics Korea; 2011 [cited 2013 Aug 20]. Available from: http://census.go.kr/hcensus/ui/ $\mathrm{html} /$ data/data_020_010_Detail.jsp?p_bitmId=60458\&q menu $=4 \& q \_s u b=2 \& q$ pageNo $=1 \& q$ div $=$ ALL .

11. Kwon IH, Lee SW. An exploratory study on reliability between on-line and off-line survey data using a single panel. J Korean Manag Rev 2005;34:1261-78.

12. Lee JY, Kim JG. Comparing data qualities of on-line panel and off-line interview surveys: reliability and validity. Korean Mark Rev 2006;21:209-31. 